\title{
Hard Competition: Stabilizing the Elusive Biaxial Nematic Phase in Suspensions of Colloidal Particles with Extreme Lengths
}

\author{
Simone Dussi, ${ }^{1, \dagger}$ Nikos Tasios, ${ }^{1}$ Tara Drwenski, ${ }^{2}$ René van Roij, ${ }^{2}$ and Marjolein Dijkstra, ${ }^{1, *}$ \\ ${ }^{1}$ Soft Condensed Matter, Debye Institute for Nanomaterials Science, \\ Utrecht University, Princetonplein 5, 3584 CC Utrecht, Netherlands \\ ${ }^{2}$ Institute for Theoretical Physics, Utrecht University, Princetonplein 5, 3584 CC Utrecht, Netherlands
}

(Received 26 January 2018; published 24 April 2018)

\begin{abstract}
We use computer simulations to study the existence and stability of a biaxial nematic $N_{b}$ phase in systems of hard polyhedral cuboids, triangular prisms, and rhombic platelets, characterized by a long $(L)$, medium $(M)$, and short $(S)$ particle axis. For all three shape families, we find stable $N_{b}$ states provided the shape is not only close to the so-called dual shape with $M=\sqrt{L S}$ but also sufficiently anisotropic with $L / S>9,11,14,23$ for rhombi, (two types of) triangular prisms, and cuboids, respectively, corresponding to anisotropies not considered before. Surprisingly, a direct isotropic- $N_{b}$ transition does not occur in these systems due to a destabilization of $N_{b}$ by a smectic (for cuboids and prisms) or a columnar (for platelets) phase at small $L / S$ or by an intervening uniaxial nematic phase at large $L / S$. Our results are confirmed by a density functional theory provided the third virial coefficient is included and a continuous rather than a discrete (Zwanzig) set of particle orientations is taken into account.
\end{abstract}

DOI: 10.1103/PhysRevLett.120.177801

Anisotropic molecules, viruses, wormlike micelles, and suspended nanoparticles can form liquid-crystal phases which exhibit long-range order of the particle orientations, possibly combined with some degree of positional order [1-3]. The simplest liquid-crystal state is the homogeneous nematic phase, which exhibits only orientational order. However, this simplicity is only apparent. For instance, the microscopic origin of the chiral nematic (cholesteric) phase and the twist-bend nematic phase is still not well understood, even though recent advances in particle synthesis [4-6], microscopy techniques, and computer simulation [7-15] have provided new insights.

A problem with an even longer history concerns the existence of a stable biaxial nematic $N_{b}$ phase, which exhibits two optical axes in contrast to the ordinary uniaxial nematic phase that displays only a single optical axis. Biaxial nematic phases have long held promise for applications in novel optoelectronic devices, but their limited window of thermodynamic stability (and for a long time even their very existence) has been of great concern. The theoretical prediction of the existence of the $N_{b}$ phase goes back to the 1970s [16,17], and first claims of its experimental observation in a micellar system date back to 1980 [18]. In 2004, novel experiments on different molecular thermotropic systems again claimed to observe the $N_{b}$ [19-22], which in the meantime was also observed in computer simulations of attractive particles [23,24]. More recently, an $N_{b}$ phase was observed in colloidal dispersions of purely repulsive boardlike particles in 2009 [25], where the stability was argued to stem from polydispersity that prevents the system from forming a smectic phase [26].
This finding in an entropy-dominated system appears to be consistent with the observation of a stable $N_{b}$ phase in early simulations of hard biaxial ellipsoids [27,28], which do not exhibit a smectic phase either [29]. Interestingly, however, recent simulations of hard spheroplatelets (with a stable smectic phase in their phase diagram) also revealed a stable $N_{b}$ phase [30], whereas ostensibly similarly shaped cuboidal particles do not [31]. On top of this confusing situation comes an unsettled issue regarding the topology of the phase diagram, in particular, whether a prolate $\left(N_{+}\right)$or oblate $\left(N_{-}\right)$uniaxial nematic phase intervenes the isotropic $(I)$ and $N_{b}$ phase or whether a direct $I-N_{b}$ phase transition is possible. According to early theoretical studies the densityshape representation of the phase diagram exhibits a cusplike feature where a rodlike regime with $I-N_{+}$coexistence and a platelike regime with $I-N_{-}$coexistence merge at the socalled dual shape into a single multicritical point with a direct $I-N_{b}$ phase transition [32,33]. More recent Landau-type theories, however, also allow for other scenarios either with or without a direct $I-N_{b}$ transition [34-37].

In this Letter, we settle the issue of the existence and stability of the biaxial nematic phase in entropy-driven systems by performing computer simulations of three different families of hard biaxial particles, extending the range of shape parameters to anisotropies much beyond hitherto considered. We see that the $N_{b}$ phase can be stable close to the dual shape, as expected, but only if the particle anisotropy exceeds a critical value which, surprisingly, varies significantly between the different particle families. In fact, we find that strong competition with the $N_{b}$ phase does not only come from the smectic phase at high densities 

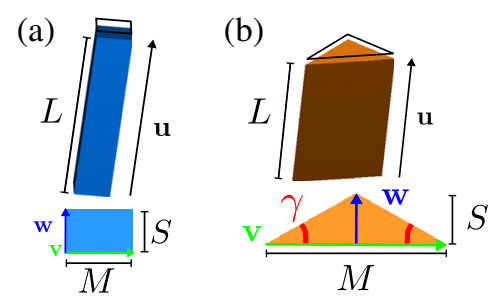

(c)

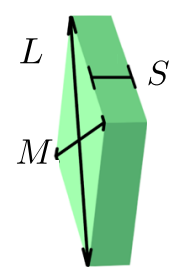

(d)

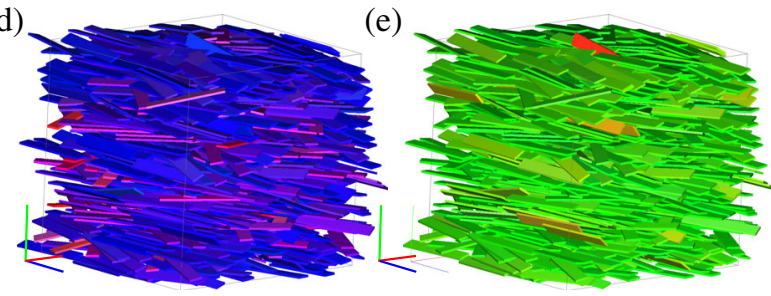

FIG. 1. Shape families of (a) cuboids, (b) triangular prisms, and (c) rhombic platelets with particle axes $L, M$, and $S$. Representative simulation snapshots (d) and (e) of a biaxial nematic $N_{b}$ phase composed of hard cuboids with $L / S=35$ and $M / S \simeq$ $5.9(\nu=0)$ at packing fraction $\eta \simeq 0.3$ of the same configuration with the particles colored according to the orientation of their (d) long or (e) short particle axis. Snapshots of an $N_{b}$ phase for triangular prisms and rhombic platelets are shown in the Supplemental Material [38].

but also from the uniaxial $N_{+}$and $N_{-}$phases at relatively low densities, such that a direct $I-N_{b}$ phase transition does not exist due to an intervening uniaxial nematic phase. The absence of this direct $I-N_{b}$ transition in our simulations is confirmed by a third-virial density functional theory with continuous rather than discrete orientations of the particles. For less anisotropic shapes, both uniaxial and biaxial nematic phases are absent from our simulations, and a direct $I$ to a positionally ordered liquid crystal phase (smectic $\mathrm{Sm}_{+}$for cuboids and prisms, columnar Col for platelets) transition is observed.

We consider the three different families of hard particles shown in Fig. 1: (a) cuboids, (b) triangular prisms, and (c) rhombic platelets, all characterized by long $(L)$, medium $(M)$, and short $(S)$ particle axes that give rise to the dimensionless particle length $L^{*} \equiv L / S$, particle width $M^{*}=M / S$, and the particle shape parameter $\nu=S / M-M / L \in[-1,1]$ —only two of which are needed to fully characterize the shape for a given family. Rodlike shapes with $\nu>0$ are expected to feature a prolate $N_{+}$ phase in their phase diagram, and platelike shapes with $\nu<0$ are expected to form an oblate $N_{-}$phase. The case $\nu=0$ [or $M=\sqrt{L S}$ or $L^{*}=\left(M^{*}\right)^{2}$ ] refers to the dual shape where biaxial nematic phases could be expected [32]. We determine the phase behavior of more than 100,60 , and 20 members, as characterized by different $L^{*}$ and $M^{*}$, of the cuboid, rhombic platelet, and triangular prism family, respectively, all as a function of packing fraction $\eta$, by performing Monte Carlo and event-driven molecular dynamics simulations of systems consisting of thousands of identical particles [38]. We show that all three families have members that exhibit a stable $N_{b}$ phase such as illustrated for cuboids with $L^{*}=35$ and $M^{*} \simeq 5.9(\nu=0)$ at $\eta \simeq 0.3$ in Fig. 1, where the same configuration is shown twice with a color coding representing the alignment of the long (d) and short (e) particle axes. We distinguish the different liquid-crystalline phases with a variety of scalar and tensorial order parameters [38].

In Fig. 2(a), we show the metaphase diagram of the family of cuboids in the $\left(M^{*}, L^{*}\right)$ plane, where the symbols represent actually simulated particle shapes, and the dashed line corresponds to the dual shape $\left(L^{*}=M^{* 2}\right.$ or $\left.\nu=0\right)$. We identify different regions according to the sequence of nematic phases as observed from low to high density beyond the isotropic phase; for clarity, we disregard the high-density smectic, columnar, and crystal phases. The black (circles) region corresponds to particle shapes that are not sufficiently anisotropic to form any nematic phase due to a direct transition from an isotropic $(I)$ to a prolate

(a)

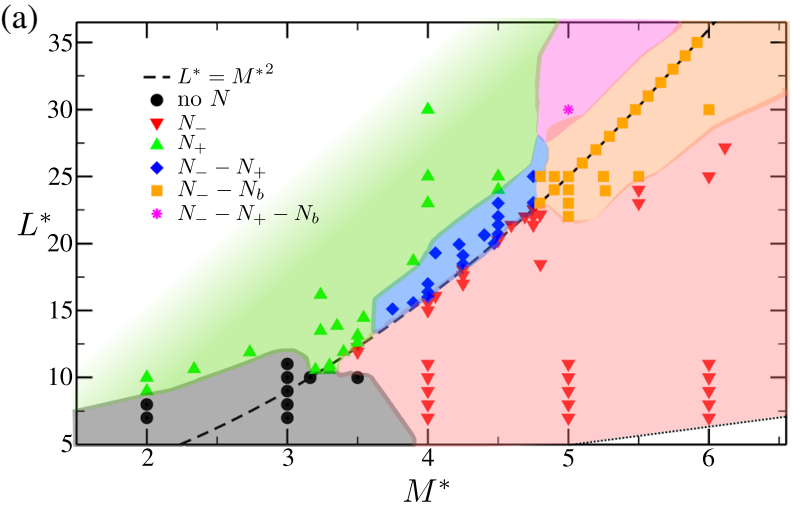

(b)

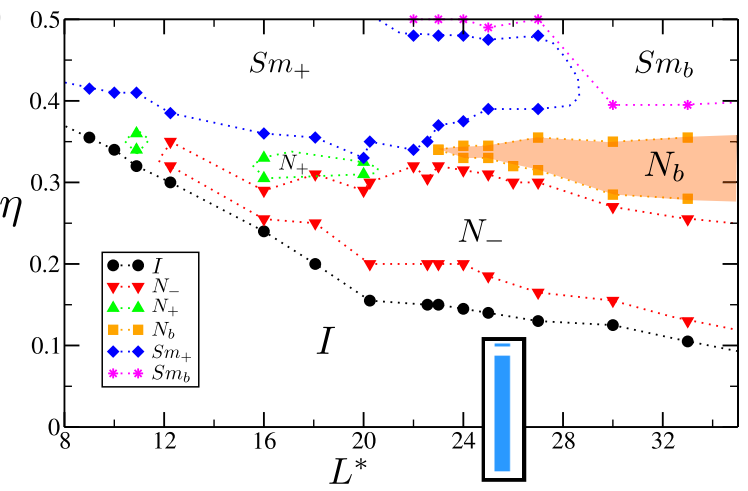

FIG. 2. (a) Metaphase diagram of hard cuboids in the dimensionless particle width and length plane spanned by $\left(M^{*}, L^{*}\right)$. Symbols denote simulated particle shapes, the dashed line indicates dual shapes $L^{*}=M^{* 2}$, and different colors denote different sequences of oblate, prolate, and biaxial nematic phases $N_{-}, N_{+}$, and $N_{b}$, respectively, upon increasing the density. (b) Phase diagram of dual-shaped cuboids in the packing fraction $L^{*}-\eta$ representation, featuring an additional isotropic phase $I$ as well as prolate and biaxial smectic phases $\mathrm{Sm}_{+}$and $\mathrm{Sm}_{b}$. The inset shows cross sections of a dual-shaped cuboid with $L^{*}=24$. 
smectic $\left(\mathrm{Sm}_{+}\right)$phase, in accordance with recent findings [31]. As expected, rodlike cuboids with $L^{*} \gg M^{* 2}$, i.e., far above the dashed line, form $N_{+}$phases (green triangles), and platelike cuboids with $L^{*} \ll M^{* 2}$, i.e., far below the dashed line, form $N_{-}$phases (red triangles). The phase sequences close to the dual shape are more interesting. At intermediate $L^{*} \sim 16-23$ and $M^{*} \sim 4-5$, in the rodlike regime above the dashed line, we find a platelike nematic $N_{-}$phase that remarkably intervenes between the $I$ and the $N_{+}$phase (blue diamonds). Surprisingly, a biaxial nematic phase only appears in Fig. 2(a) for cuboids close to the dual shape when particle anisotropies are as extreme as $L^{*}>23$ and $M^{*}>5$, again with an intervening $N_{-}$phase (orange squares). Quite deep in the rodlike regime, $L^{*}>30$ and $M^{*}>5$, we even observe an intricate $N_{-}-N_{+}-N_{b}$ phase sequence (pink asterisks). Hence, the nematic phase in coexistence with the isotropic phase is $N_{-}$in a substantial region even where $L^{*}>M^{* 2}$ (or $\nu>0$ ). No direct $I-N_{b}$ transition is found for cuboidal particles. This conclusion is even more apparent in Fig. 2(b), where we present the phase diagram of dual-shaped cuboids in the $L^{*}-\eta$ representation, where the lines denote the approximate binodals. For $L^{*}<11$, we observe the strongly first-order phase transition
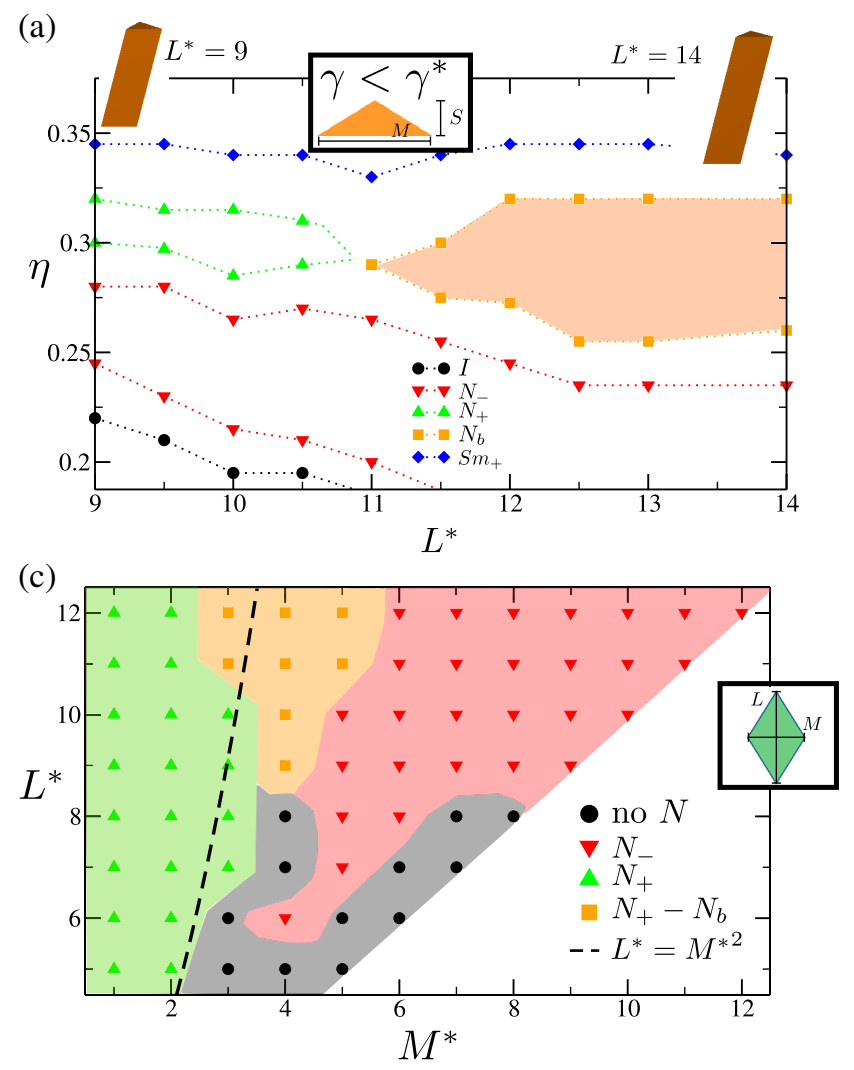

from the $I$ to a prolate smectic $\left(\mathrm{Sm}_{+}\right)$phase. Furthermore, Fig. 2(b) reveals for $L^{*}>12$ that the coexistence with the isotropic phase is solely with the $N_{-}$phase, which is stable for a surprisingly large density regime before it transforms into an $N_{b}$ phase for $L^{*}>23$ and into $N_{+}$for $16<L^{*}<20$. The $N_{b}$ phase is seen to be stable for a wider range of $\eta$ upon increasing $L^{*}$. Compressing the $N_{b}$ to the smectic phase leads to biaxial order being lost unless $L^{*}>28$.

Our present study extends significantly beyond previous simulations on cuboids with $M^{*}=1$ and $0.125 \leq L^{*} \leq 5$, where no biaxial order was found nor expected [50]. In the search for a biaxial nematic phase, Cuetos et al. [31] recently performed extensive simulations of more anisotropic cuboids with $L^{*}=9,12$ and $1 \leq M^{*} \leq L^{*}$, but an $N_{b}$ phase was not observed, consistent with our finding that $L^{*}>23$ is required. Interestingly, dual-shaped cuboids with $L^{*}=23$ have $L / M=4.8$, which is large enough for uniaxial rods to form a stable nematic phase [51], whereas $L^{*}=12$ only yields $L / M=3.5$ which does not suffice.

We now turn our attention to the phase behavior of triangular prisms with a dual shape $\left(L^{*}=M^{* 2}\right)$ and an isosceles triangular base with a base angle $\gamma$ as defined in Fig. 1(b). In Fig. 3, we present the phase diagram of these
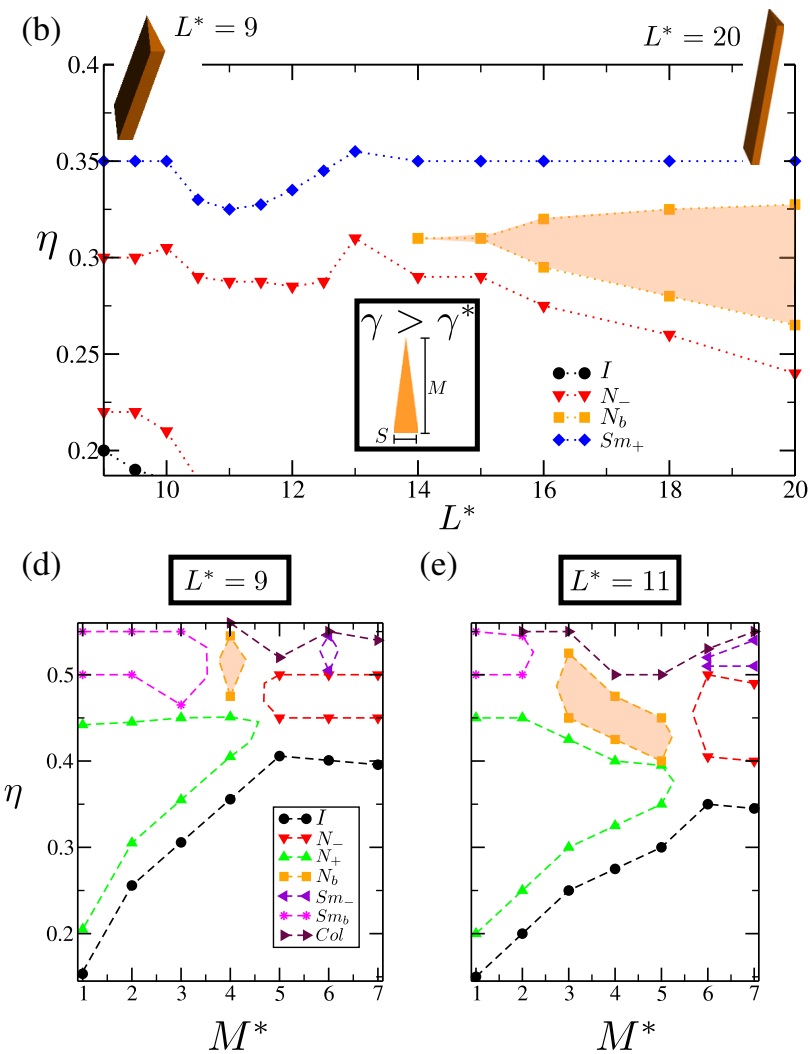

FIG. 3. Phase diagram of dual-shaped triangular prisms in the $L^{*}-\eta$ representation for (a) $\gamma<\gamma^{*}=\pi / 3$ and (b) $\gamma>\gamma^{*}$, see inset and text. The dashed lines denote the binodals. (c) Metaphase diagram of hard rhombic platelets in the $\left(M^{*}, L^{*}\right)$ plane. Symbols denote simulated particle shapes, the dashed line indicates dual shapes $L^{*}=M^{* 2}$, and different colors denote different sequences of nematic phases upon increasing the density. Phase diagram of hard rhombic platelets in the $M^{*}-\eta$ representation for (d) $L^{*}=9$ and (e) $L^{*}=11$, featuring an oblate smectic $\mathrm{Sm}_{-}$phase and columnar Col phase. 
particles in the $L^{*}-\eta$ representation, in (a) for $\gamma<\gamma^{*}$ and in (b) for $\gamma>\gamma^{*}$, where $\gamma^{*}=\pi / 3$ is the cross-over angle between one large and two small sides of the triangular base for $\gamma<\gamma^{*}$ to vice versa for $\gamma>\gamma^{*}$ (see insets). Both phase diagrams display an $N_{-}-N_{b}$ phase sequence for sufficiently large particle anisotropies $L^{*} \geq 11$ in (a) and $L^{*} \geq 14$ (b), i.e., without any direct $I-N_{b}$ transition due to an intervening $N_{-}$phase. For smaller $L^{*}$, we observe an $N_{-}-N_{+}-\mathrm{Sm}_{+}$phase sequence in (a), whereas this intervening $N_{+}$phase is absent in (b).

Next, we consider in Fig. 3(c) the metaphase diagram of the family of rhombic platelets in the $M^{*}-L^{*}$ plane (with $L^{*}>M^{*}$ ), with a focus on the sequence of nematic states upon increasing the density. The dual shape is represented by the dashed line. We again observe $N_{+}$for $L^{*} \gg M^{* 2}$ and $N_{-}$for $L^{*} \ll M^{* 2}$, but now with prolate order invading the region $\nu<0$. A striking feature is the appearance of an $I-N_{+}-N_{b}$ sequence (orange squares), not only above the dashed line but also below it in the "platelike" regime. As for cuboids and triangular prisms, we also find that the family of rhombic platelets does not display a direct $I-N_{b}$ transition. This is also evident from the $M^{*}-\eta$ phase diagrams for $L^{*}=9$ and $L^{*}=11$ shown in Figs. 3(d) and 3(e), respectively. Figures 3(d) and 3(e) also show that the $N_{b}$ phase transforms into a columnar phase Col upon increasing the density instead of a smectic phase in the case of cuboids and triangular prisms.

In order to shed further light on the subtleties involved in the absence of a direct $I-N_{b}$ transition due to the intervening $N_{-}$phase in the case of biaxial cuboids, we also employed density functional theory for these particle shapes. We consider both a second- and a third-virial theory, not only for the Zwanzig model with six discrete particle orientations [26,52] but also for cuboids with a continuum of particle orientations treated within an expansion into Wigner matrices [38]. Within a second-virial theory, there is perfect symmetry of prolate and oblate ordering with respect to the dual shape. Contrary to Mulder's conclusion on the basis of a bifurcation analysis [32], our numerical free-energy minimizations yield no direct $I-N_{b}$ transition, at least for dual-shaped Zwanzig cuboids with $L^{*}>11$, but rather an $I-N_{+} / N_{-}-N_{b}$ sequence, where the $N_{+} / N_{-}$phases have identical free energies within the second-virial theory, see Fig. 4(a). However, in agreement with [26], we do find a direct $I-N_{b}$ transition for $L^{*} \leq 11$. In the third-virial theory for the Zwanzig model, the $N_{+} / N_{-}$symmetry is broken in favor of $N_{+}$at $\nu=0$ as shown in Fig. 4(b), in agreement with the fundamental measure theory results of Ref. [52] but not in agreement with our simulations of the continuum model, where $N_{-}$is favored. Applying the second-virial theory to the continuum model restores the symmetry about $\nu=0$ as illustrated in the phase diagram of cuboids with $M^{*}=8$ in the $L^{*}-\eta$ representation in Fig. 4(c), yet again in disagreement with our simulations, where the symmetry (a)

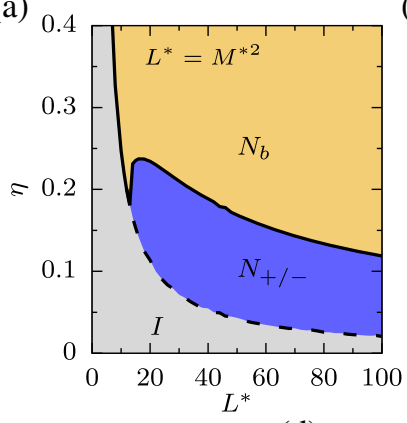

(b)

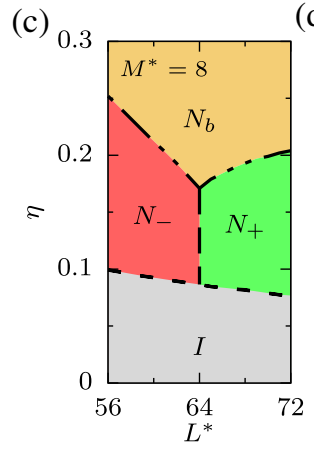

(d)


FIG. 4. Phase diagram for dual-shaped cuboids in the $L^{*}-\eta$ plane for a Zwanzig model within the (a) second- and (b) thirdvirial theory. (c) Phase diagram for $M^{*}=8$ cuboids in the $L^{*}-\eta$ plane within the full-orientation second-virial theory. (d) Metaphase diagram of cuboids in the $\left(M^{*}, L^{*}\right)$ plane for continuum orientations within a full third-virial theory. Different symbols denote different nematic phase sequences from low to high packing fraction (up to $\eta=0.6$ for $L^{*}<50$ and up to $\eta=0.4$ for $L^{*} \geq 50$ ), neglecting spatially ordered phases.

is broken. Only for a continuum of orientations combined with the third-virial approximation is qualitative agreement with the simulations obtained, since then the phase sequence $I-N_{-}-N_{b}$ appears for $\nu=0$. The resulting metaphase diagram of the family of hard cuboids in the $\left(M^{*}, L^{*}\right)$ plane is shown in Fig. 4(d), which clearly shows that the $N_{-}$phase invades the rodlike regime above the dashed line that represents the dual shape.

In conclusion, for three large families of biaxial particle shapes, we have shown that the $N_{b}$ phase can be stable close to the dual shape but only if the particle shape is sufficiently anisotropic with $L^{*}>L_{\min }^{*}=9,11,14,23$ for rhombi, prisms with angle $\gamma<\pi / 3$ and $\gamma>\pi / 3$, and cuboids, respectively. Interestingly, for dual-shaped spheroplatelets that closely resemble our cuboids at first sight, recent simulations [30,53] revealed an $N_{b}$ phase for $L^{*}>9$ rather than $L^{*}>23$ for cuboids. This trend in which particle shapes with triangular, rounded, or rhombic cross sections have smaller $L_{\min }^{*}$ than cuboids suggests that the 2D packing efficiency of the particle cross section in a smectic layer largely determines $L_{\text {min }}^{*}[31,33,54,55]$. Rhombic platelets with their minimal $L_{\min }^{*}$ are indeed ideal candidates for observing an $N_{b}$ phase. Finally, none of the 
members of the different shape families exhibit a direct $I-N_{b}$ transition due to the surprising stability of an intervening uniaxial nematic phase. Future experimental efforts towards the self-assembly of biaxial colloids will benefit from these results, with the knowledge that imperfections, such as roundness, facet corners, or polydispersity in particle shape can facilitate the self-assembly of biaxial nematic phases.

This work is part of the D-ITP consortium, a program of the Netherlands Organization for Scientific Research (NWO) that is funded by the Dutch Ministry of Education, Culture and Science. We acknowledge financial support from an NWO-ECHO and an NWO-VICI grant. We thank Bela Mulder for helpful discussions and Oleg Lavrentovich for useful comments about experiments on thermotropic systems.

S. D., N. T., and T. D. contributed equally to this work.

”m.dijkstra@uu.nl

Present address: Physical Chemistry and Soft Matter, Wageningen University, Stippeneng 4, 6708 WE, Wageningen, Netherlands.

[1] L. Onsager, Ann. N.Y. Acad. Sci. 51, 627 (1949).

[2] W. Maier and A. Saupe, Z. Naturforsch. A 14, 882 (1959).

[3] P.-G. de Gennes and J. Prost, The Physics of Liquid Crystals (Oxford University Press, Cambridge, England, 1993).

[4] Y. Xia, Y. Xiong, B. Lim, and S. Skrabalak, Angew. Chem., Int. Ed. Engl. 48, 60 (2009).

[5] J. Gong, G. Li, and Z. Tang, Nano Today 7, 564 (2012).

[6] L. Zhang, W. Niu, and G. Xu, Nano Today 7, 586 (2012).

[7] U. Agarwal and F. A. Escobedo, Nat. Mater. 10, 230 (2011).

[8] P. F. Damasceno, M. Engel, and S. C. Glotzer, Science 337, 453 (2012).

[9] L. Mederos, E. Velasco, and Y. Martinez-Raton, J. Phys. Condens. Matter 26, 463101 (2014).

[10] H. Kolli, E. Frezza, G. Cinacchi, A. Ferrarini, A. Giacometti, T. Hudson, C. De Michele, and F. Sciortino, Soft Matter 10, 8171 (2014).

[11] C. Greco and A. Ferrarini, Phys. Rev. Lett. 115, 147801 (2015).

[12] A. Kuhnhold and T. Schilling, J. Chem. Phys. 145, 194904 (2016).

[13] Š. Rủžička and H. H. Wensink, Soft Matter 12, 5205 (2016).

[14] M. Dijkstra, Adv. Chem. Phys. 156, 35 (2015).

[15] S. Dussi and M. Dijkstra, Nat. Commun. 7, 11175 (2016).

[16] M. J. Freiser, Phys. Rev. Lett. 24, 1041 (1970).

[17] J. P. Straley, Phys. Rev. A 10, 1881 (1974).

[18] L. J. Yu and A. Saupe, Phys. Rev. Lett. 45, 1000 (1980).

[19] G. R. Luckhurst, Nature (London) 430, 413 (2004).

[20] K. Severing and K. Saalwächter, Phys. Rev. Lett. 92, 125501 (2004).

[21] L. A. Madsen, T. J. Dingemans, M. Nakata, and E.T. Samulski, Phys. Rev. Lett. 92, 145505 (2004).

[22] B. R. Acharya, A. Primak, and S. Kumar, Phys. Rev. Lett. 92, 145506 (2004).
[23] R. Berardi and C. Zannoni, J. Chem. Phys. 113, 5971 (2000).

[24] R. Berardi, L. Muccioli, S. Orlandi, M. Ricci, and C. Zannoni, J. Phys. Condens. Matter 20, 463101 (2008).

[25] E. van den Pol, A. V. Petukhov, D. M.E. Thies-Weesie, D. V. Byelov, and G. J. Vroege, Phys. Rev. Lett. 103, 258301 (2009).

[26] S. Belli, A. Patti, M. Dijkstra, and R. van Roij, Phys. Rev. Lett. 107, 148303 (2011).

[27] M. P. Allen, Liq. Cryst. 8, 499 (1990).

[28] P. J. Camp and M. Allen, J. Chem. Phys. 106, 6681 (1997).

[29] D. Frenkel, B. M. Mulder, and J. P. McTague, Phys. Rev. Lett. 52, 287 (1984).

[30] S. D. Peroukidis and A. G. Vanakaras, Soft Matter 9, 7419 (2013).

[31] A. Cuetos, M. Dennison, A. Masters, and A. Patti, Soft Matter 13, 4720 (2017).

[32] B. Mulder, Phys. Rev. A 39, 360 (1989).

[33] M. P. Taylor and J. Herzfeld, Phys. Rev. A 44, 3742 (1991).

[34] L. Longa and G. Pająk, Liq. Cryst. 32, 1409 (2005).

[35] D. Allender and L. Longa, Phys. Rev. E 78, 011704 (2008).

[36] P. K. Mukherjee and K. Sen, J. Chem. Phys. 130, 141101 (2009).

[37] G. R. Luckhurst and T. J. Sluckin, Biaxial Nematic Liquid Crystals: Theory, Simulation and Experiment (John Wiley \& Sons, New York, 2015).

[38] See Supplemental Material at http://link.aps.org/ supplemental/10.1103/PhysRevLett.120.177801 for details on simulation and theoretical methods and additional results, which includes Refs. [39-49].

[39] N. Tasios and M. Dijkstra, J. Chem. Phys. 146, 144901 (2017).

[40] N. Tasios, Ph. D. thesis, Utrecht University, 2017.

[41] GAMMA Research Group, RAPID—Robust and Accurate Polygon Interference Detection, http://gamma.cs.unc.edu/ $\mathrm{OBB} /$.

[42] E. Gilbert, D. Johnson, and S. Keerthi, IEEE Trans. Rob. Autom. 4, 193 (1988).

[43] G. van den Bergen, J. Graphics Tool 4, 7 (1999).

[44] R. Rosso, Liq. Cryst. 34, 737 (2007).

[45] B. M. Mulder, Mol. Phys. 103, 1411 (2005).

[46] S. Belli, S. Dussi, M. Dijkstra, and R. van Roij, Phys. Rev. E 90, 020503(R) (2014).

[47] S. Dussi, S. Belli, R. van Roij, and M. Dijkstra, J. Chem. Phys. 142, 074905 (2015).

[48] D. Frenkel, J. Phys. Chem. 91, 4912 (1987).

[49] D. Frenkel, J. Phys. Chem. 92, 5314 (1988).

[50] B. S. John, C. Juhlin, and F. A. Escobedo, J. Chem. Phys. 128, 044909 (2008).

[51] P. Bolhuis and D. Frenkel, J. Chem. Phys. 106, 666 (1997).

[52] Y. Martínez-Ratón, S. Varga, and E. Velasco, Phys. Chem. Chem. Phys. 13, 13247 (2011).

[53] S. D. Peroukidis, A. G. Vanakaras, and D. J. Photinos, Phys. Rev. E 88, 062508 (2013).

[54] Y. Martínez-Ratón, S. Varga, and E. Velasco, Phys. Rev. E 78, 031705 (2008).

[55] M. Marechal, S. Dussi, and M. Dijkstra, J. Chem. Phys. 146, 124905 (2017). 